\title{
Engine knock margin estimation using in-cylinder pressure measurements
}

\author{
Giulio Panzani, Fredrik Östman and Christopher H. Onder
}

\begin{abstract}
Engine knock is among the most relevant limiting factors in the improvement of the operation of spark ignited engines. Due to an abnormal combustion inside the cylinder chamber, it can cause performance worsening or even serious mechanical damage. Being the result of complex local chemical phenomena, knock turns out to have a significant random behaviour but the increasing availability of new on-board sensors permits a deeper understanding of its mechanism. The aim of this paper is to exploit in-cylinder pressure sensors to derive a knock estimator, based on the logistic regression technique. Thanks to the proposed approach it is possible to explicitly deal with knock random variability and to define the so-called margin (or distance) from the knocking condition, which has been recently proven to be an effective concept for innovative knock control strategies. In a model-based estimation fashion, two modelling approaches are compared: one relies on well-known physical mechanisms while the second exploits a principal component analysis to extract relevant pressure information, thus reducing the identification effort and improving the estimation performance.
\end{abstract}

Index Terms-Engine knock estimation, knock control, incylinder pressure sensors, logistic regression, principal component analysis

\section{NOMENCLATURE}

\section{A. Acronyms}

SI Spark ignition.

SA Spark advance.

MAPO Maximum Amplitude of Pressure Oscillations, used to detect an engine knocking cycle.

$\boldsymbol{K M}$ Knock Margin: quantifies the distance of a considered cycle from the knocking conditions.

KOCA Knock Onset Crank Angle: crank angle value where the knock event is detected.

IVC Intake Valve Closing.

PSO Particle Swarm Optimization.

AFR Air-to-Fuel Ratio.

PCA Principal Component Analysis.

SVD Singular Value Decomposition.

This project has received funding from the European Union's Horizon 2020 research and innovation programme under grant agreement No 634135.

G. Panzani is with the Dipartimento di Elettronica, Informazione e Bioingegneria, Politecnico di Milano, 20133 Milano, Italy (e-mail: giulio.panzani@polimi.it).

C. H. Onder is with the Institute of Dynamic Systems and Control, Department of Mechanical and Process Engineering, Swiss Federal Institute of Technology (ETH), 8092 Zürich, Switzerland.

F. Östman is with Wärtsilä Finland Oy, FIN-65101, Vaasa, Finland.

\section{B. Symbols}

$R_{L}^{2} \quad$ Pseudo- $R^{2}$ index for knock estimation quality

evaluation. $[-]$

$\hat{C} \quad$ Hosmer and Lemeshow index for knock estima-

tion quality evaluation. [-]

$\tau \quad$ Self-ignition delay of unburned mixture. [s]

$p \quad$ In-cylinder pressure. [bar]

$T \quad$ In-cylinder temperature. [K]

$T_{i m} \quad$ Intake manifold temperature. $\quad[\mathrm{K}]$ or $[\mathrm{C}]$

$\omega \quad$ Engine speed. [rpm]

$\theta \quad$ Crank angle. [deg]

$\lambda \quad$ Air-to-fuel ratio. [-]

$C_{1}, C_{2}, C_{3}$ Arrhenius-like self-ignition model coefficients.

$[-],[-],[-]$

$\bar{p}_{1,2, \ldots, i} \quad 1,2, \ldots, i$-th eigenpressure. [bar]

$\gamma_{1,2, \ldots, i} \quad 1,2, \ldots, i$-th eigenpressure coefficient used to describe the pressure trace of each engine cycle. [-]

\section{Subscripts}

$k \quad$ referred to the $k$-th engine cycle.

$g b \quad$ referred to the grey-box approach.

phy referred to the physical approach.

\section{INTRODUCTION AND MOTIVATION}

$\mathbf{T}$ $\mathrm{HE}$ combustion process in SI engines is normally triggered by the spark, whose timing is accurately defined in order to achieve the desired engine performance. In particular engine operating conditions, a too early spark timing may cause an abrupt unburned mixture (end-gas) self-ignition, due to the high temperature and pressure conditions reached inside the cylinder chamber. This event is usually termed knock, recalling the typical metallic sound caused by the shock waves generated by the spontaneous detonation of the air/fuel mixture. Such event limits the improvement of engine performance, being responsible for some undesirable effects: while it can cause serious cylinder damages, less dramatic consequences are powertrain oscillations, a general decrease of engine efficiency and an increase of pollutant emissions [33]. An accurate control of SI timing has thus lately become a crucial issue in the development of advanced combustion control systems.

In the scientific literature specific attention has been paid to the knock event due to its applicative relevance. The air/fuel self ignition is the result of complex local phenomena in the 
cylinder chamber and as such shows significant experimental random nature. For this reason, the first research efforts have been devoted mainly to knock sensing and detection [32], [16], with the development of techniques and technologies that, flanked with efficient signal processing algorithms, could be able to reliably discriminate knocking from not knocking cycles (and, if possible, to quantify the detonation severity).

Knock control strategies developed consequently: the standard approach, which has been widely adopted in series production, can be classified as event-based where, based on simple [17] or more complex [30] rules, a single measured knock occurrence causes a controller intervention. In order to cope with knock random nature, stochastic knock control strategies have been recently proposed. Their main idea is to compare the statistic knock properties of the current engine operating point (rather than considering each event individually) with a target value and to adapt the control action accordingly. In [24] the feedback statistic is established as a cumulative sum of knocking events over a certain number of cycles, whereas in [25] a likelihood ratio approach is employed. In [8] a nonlinear transformation is used to shape the random distribution of the knock events as a Gaussian variable whose mean and variance are recursively estimated and used as feedback signals for the knock control strategy. The advantage of stochastic approaches is the fact that reckoning with the stochastic knock behaviour leads to better mean engine running conditions and to less cyclic variability. The drawback of the mentioned strategies lays in the fact that the feedback statistic signal is built in real-time, which requires several cycles. Given a single engine cycle, out of the current operating point history, no statement about the expected knock rate is possible.

The control strategy proposed by Lezius et al. in [18] approaches the problem differently. It is based on the evidence that cycles with a higher peak pressure are more likely to knock. Engine knock is thus closed-loop regulated tracking a peak pressure reference that is a compromise between engine output torque and engine knock tendency. The distinguishing feature of this approach is the fact that a margin (or distance) from the knocking condition is defined for any single cycle. In this specific case the cycle peak pressure is used to estimate engine knock and its distance, computed as the error between the measured and the target peak pressure value.

Proper models are required to design such a knock margin estimator. In Lezius' work, the model is implicitly enclosed in the experimental evidence of a more frequent knock occurrence for higher peak pressure cycles. With respect to the realtime stochastic approaches described previously, the additional modelling effort compensates for the advantage of a cycle-tocycle knock margin evaluation. While the work of Lezius et al. is focused primarily on the development of the control concept, the knock estimator there employed has certain shortcomings:

- The proposed experimental correlation between knock and cycle peak pressure is map-based; a parametric estimator would be preferable to a non-parametric one.

- The knock margin estimator employed has no relationship with any statistical characterization of knock; the target peak pressure, thus, cannot be easily defined.

- The peak pressure correlation with knock occurrence, even though valid and physically justified, is too restrictive; to provide a reliable model in all engine operating conditions other factors which significantly affect knock tendency (see [33]) should be taken into account.

Knock modelling is a widely discussed topic in literature, where numerous solutions can be found. These can be sorted in terms of complexity: starting from 3D CFD fluid dynamic simulations coupled with detailed auto-ignition chemistry reactions, ending to 0D gas models where empirical formulations based on Arrhenius functions provide a lumped parameter description of the complex chemical reaction involved, see e.g. [19], [7]. These solutions, even in their easiest formulations are not suited for real-time applications due to their high computational and/or tuning cost. Moreover, despite some papers [28], [9] deal with the cycle-to-cycle combustion variations (which represents only one contribution to knock variability), traditional knock models are deterministic and are thus generally used in engine design and sensitivity analysis rather than for control.

Pushed by the promising results of the knock margin control strategy, the goal of the present work is to overcome the limitations listed, by proposing a model-based knock estimator that can be used in engine control applications. The technological feature at the basis of this study is the availability of in-cylinder pressure sensors measures. In-cylinder pressure sensors provide a direct insight into the combustion phenomena since they provide the measure of the pressure in a specific location of the cylinder chamber. So far, they have been employed in prototype or research activities but their currently decreasing cost has made their use feasible even for series production applications.

In the first part of the paper (Section II) an overview on engine knock is given, introducing knock detection and knock margin concepts based on in-cylinder pressure measurements. The knock estimation approach, along with the knock margin evaluation, is set in Section III. Knock random nature inclusion in the modelling process is also addressed: eventually, the knock occurrence probability is modelled as function of engine measurements, using pattern recognition/machine learning modelling techniques, which is one of the distinguishing feature of the proposed estimator. To account for the various conditions of several engine operating points two competing estimators are proposed in Section IV. On the one hand a physics-based approach, inspired by knock modelling literature, is used. On the other hand, a principal component analysis (PCA) extracts the relevant information from engine measurement data, with a genuine grey-box estimation fashion. The competing approaches comparison is drawn in Section V, based on experimental data collected in a wide range of engine operating conditions. Some final remarks conclude the paper.

\section{ENGINE KNOCK AND IN-CYLINDER PRESSURE SENSOR}

\section{A. Knock event and knock margin}

A knock event is characterised by the abnormal combustion of the unburned gas, that causes high temperatures, high pressure peaks and an acoustic resonance within the cylinder. Knock detection is based on such features and it 
has been investigated intensively in order to obtain a knockintensity/knock occurrence metric to be used for its control. Indeed, in-cylinder pressure waves show a characteristic and constant frequency that can be estimated with good approximation given the chamber geometric specifications, see [1]; a band-pass filtered pressure signal can be thus used to detect knock and to quantify its intensity. As an example, Figure 1 shows the pressure signals of two different cycles, along with the corresponding band-pass filtered versions in the lower panel. The maximum amplitude of pressure oscillation
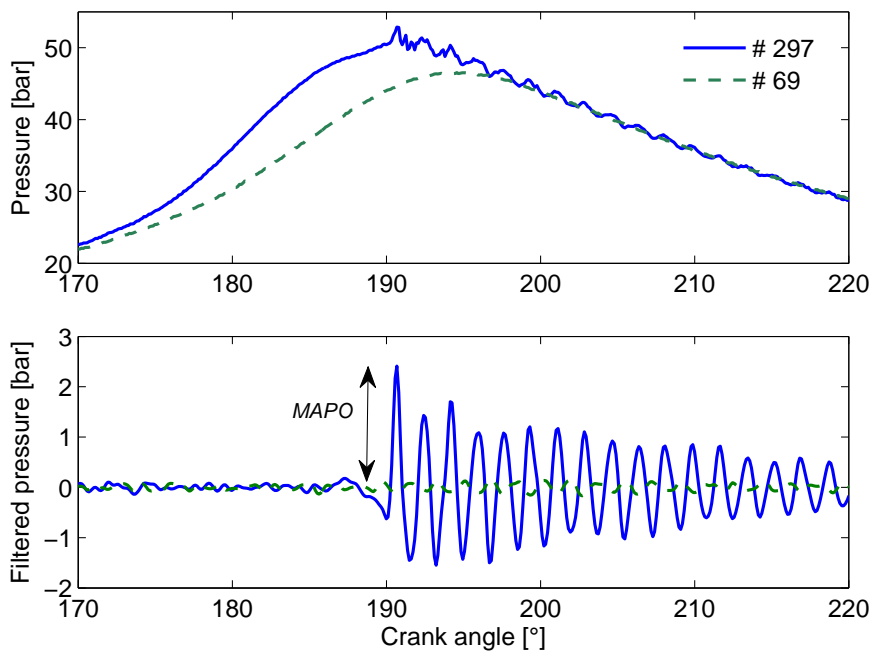

Fig. 1. Pressure and band-bass filtered signal for knock detection and knock intensity definition.

$(M A P O)$ index is commonly used to characterize knock intensity. Based on this index, a knock/not-knock classification of each $k$-th cycle can be established:

$$
\begin{aligned}
& y(k)=1 \quad \text { if } M A P O(k) \geq \delta \quad \Leftrightarrow \quad \text { knocking cycle } \\
& y(k)=0 \text { if } \operatorname{MAPO}(k)<\delta \quad \Leftrightarrow \quad \text { not-knocking cycle }
\end{aligned}
$$

where $\delta$ is a pre-determined threshold.

Although the in-cylinder pressure defines the current engine knocking state, a still open issue, especially for not-knocking situations, is the ability to evaluate how close is a current operating condition to a knocking one. This is the core of the so-called knock margin index which is designed to quantify such a proximity condition. Knock margin based control strategies exploit such information, trying to regulate the engine operation on the verge of knocking conditions.

To provide a better understanding of the margin concept, Figure 2 shows three different pairs of engine cycles, each corresponding to a different value of spark advance (SA) angle. As revealed by pressure oscillations the highest pressure couple shows a clear knocking behaviour, whereas the lower pressure traces a not-knocking one. The knock margin problem occurs when the dashed lines are considered: both cycles are not knocking but clearly the higher pressure cycle, indicated by the lighter line, is closer to a knocking condition than the other and, thus, it should be treated as a warning situation. The plots in the right-hand panel show the corresponding band-pass filtered pressure for the dashed cycles. Although this kind of
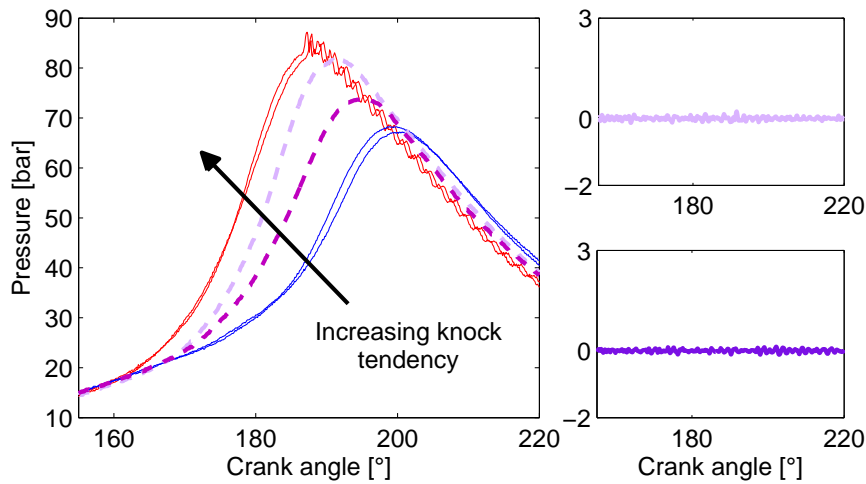

Fig. 2. Knock margin concept illustrated via pressure profiles of various operating points.

signal is efficiently employed for the detection of knock events, it cannot provide any useful indication about the distance (or margin) from the knocking conditions.

The knock margin estimation problem can be split into two sub-problems: on the one side, defining which engine conditions lead to a knocking cycle; on the other finding a procedure to define the distance of the actual from the critical conditions.

The availability of in-cylinder pressure allows an enginetechnology independent approach to the knock margin estimation. Different engine technologies (e.g. engine turbocharger presence, direct or port fuel injection, inter-cooler presence) can be seen as a cause/factor that influence the combustion process, tightly related to the knock events. However, the direct access to the chamber pressure allows to gather all the necessary information on the combustion event, overlooking at the cause that has produced it.

\section{B. A stochastic interpretation for knock and knock margin}

The intrinsic random nature of knock is a widely accepted fact, see e.g. [25], [8]. On one side, the cycle-to-cycle variability in the combustion causes pressure and temperature variations inside the cylinder chamber, thus leading to different cycle knocking behaviour. The upper panel of Figure 3 shows an example of such variations, providing the pressure traces of a steady-state operating point. If cycle-to-cycle variability were the only factor responsible for the knock stochastic behaviour, it would be possible to deterministically link any measured pressure traces with a knocking behaviour. Unfortunately, this is not the case. On the lower panel of Figure 3 the pressure traces of two cycles extracted from the same engine operating condition are shown. Although the pressure traces are identical - at least the pressure differences are well below the variation expected from cycle-to-cycle - knock occurs in one case, whereas not in the other.

This peculiarity brings to re-interpret the knock margin estimation: since there is no deterministic way to link engine conditions to knock, a knock estimator cannot provide a knock/not knock statement, but rather an indication about its probability. Within the same statistical perspective, the knock margin must be seen not as the distance from knock conditions, but as the distance from an engine knock probability (defined as critical for the considered application). 

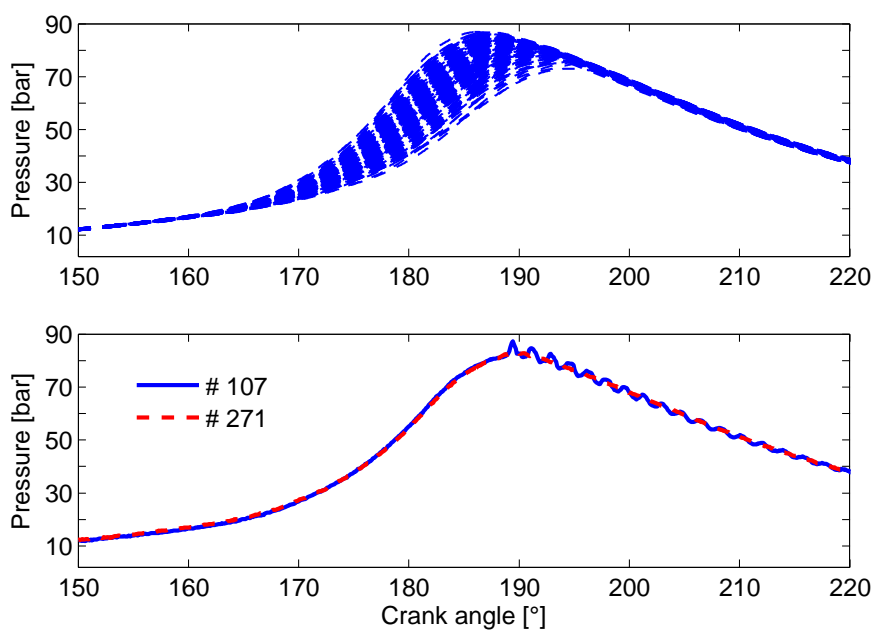

Fig. 3. Cycle-to-cycle variability in a steady-state operating point (upper panel) and example of significantly different knock behaviours for two similar engine cycles (lower panel).

\section{MODEL-BASED KNOCK MARGIN ESTIMATION}

To solve the knock margin estimation problems, a modelbased approach is here presented: a model which describes the relationship between the in-cylinder pressure and the cycle knock probability is identified; a geometric interpretation of such model makes the knock margin definition possible. Fed by the data describing an engine cycle, the identified model can be then used in real-time engine control applications to estimate the knock probability and margin.

Finding proper correlations among engine measures and engine knock is thus the key issue of the proposed estimator. It is here assumed that there is no dynamic in the inquired model, i.e. that knock of a given cycle depends only on the current pressure trace, rather than on the previous cycles history. Despite this assumption simplifies the modelling problem reducing it to a static regression between the input and the output variables, some issues must be still properly addressed. Firstly the output measured variable $y(k)$, which has to be used to train and validate the model, is binary and not continuous. Moreover, the engine knock model should allow to easily define a procedure to compute the distance from critical conditions. Finally as already recalled, a probabilistic interpretation of the estimated margin is a desirable (if not mandatory) feature.

In the following, a machine learning modelling approach is presented which allows to efficiently provide a solution for the above-mentioned points.

\section{A. Logistic regression as modelling approach}

Knock detection is the result of a classification process (1) and as such, knock occurrence is naturally described by a binary signal. Considering this aspect, it comes natural to pose the knock modelling as a classification problem as well, i.e. to define a procedure that, given a certain number of features describing an observed event, allows it to be assigned to a known class. There exists a great variety of classification algorithms, that can be sorted according to various characteristics. A good review can be found in [13]. In the present work, the so-called logistic regression [12] is used.

For each cycle, also called instance, there are $q=2$ possible classes: knocking or not-knocking. An instance is also described by $n$ numerical attributes $x_{1}, x_{2}, x_{3} \ldots x_{n}$ (that somehow carry the in-cylinder pressure information), that can be collected in the attribute or feature vector $\mathrm{x}$. Thus, each instance can be represented as a point in a n-dimensional feature space and has associated a label $q$.

As some other (not all) classification techniques do, the logistic regression defines a hyper-surface which aims at the best possible separation of instances belonging to different classes. The resulting surface divides the feature hyper-space in two regions, each referring to one of the possible classes. The classification of a new instance is made by checking its related point position with respect to the hyper-surface, which can be interpreted as a way to describe all the critical conditions which lead to knock. Considering this geometric interpretation of the classification process, the general advantage of such an idea is clear: it is indeed straightforward to introduce a margin concept, defined as geometric distance of the instance point from the classification hyper-surface.

The hyper-surface classification approach is shared by other well known procedures, such as the Support Vector Machine (SVM), Fischer Linear and the Quadratic discriminant classification. Among the mentioned alternatives, logistic regression has been preferred because it permits to handle the statistical nature of the phenomena easily, providing the necessary probability interpretation of the knock margin model. In fact, this classification algorithms explicitly link the probability of the two possible events to the attribute values $\mathbf{x}$, using the logistic function:

$$
\begin{array}{ll}
P(y=1 \mid \mathbf{x})=\pi(\mathbf{x}) & =\frac{e^{(\alpha+\boldsymbol{\beta} \mathbf{x})}}{1+e^{(\alpha+\boldsymbol{\beta} \mathbf{x})}} \\
P(y=0 \mid \mathbf{x})=1-\pi(\mathbf{x}) & =\frac{1}{1+e^{(\alpha+\boldsymbol{\beta} \mathbf{x})}}
\end{array}
$$

The scalar $\alpha$ and the vector $\boldsymbol{\beta}$ are the logistic model parameters, that have to be estimated. Another appealing property of the logistic regression is that, according to equation (2), the $\log$-odds $\Lambda(\mathbf{x})$ is a linear function of the model parameters:

$$
\log \Lambda(\mathbf{x})=\log \left(\frac{\pi(\mathbf{x})}{1-\pi(\mathbf{x})}\right)=\alpha+\boldsymbol{\beta} \mathbf{x} .
$$

This linear fashion of the logistic regression eases the interpretation of modelling results and suggests its link to the linear regression approach.

Recalling the classification geometric interpretation, the hypersurface marks a limit condition in which the classification it is not possible. In probabilistic terms, this means that the probability of two events is identical $(\pi(\mathbf{x})=1-\pi(\mathbf{x})=0.5)$. This situation, according to equation (3), leads to:

$$
\log \Lambda(\mathbf{x})=0 \Rightarrow \alpha+\boldsymbol{\beta} \mathbf{x}=0 .
$$

Such equation completely describes the classification hypersurface as an hyper-plane $(\epsilon)$ in the feature space:

$$
\epsilon: \alpha+\boldsymbol{\beta} \mathbf{x}=0 \text {. }
$$


If the $k$-th cycle is represented as a point $\sigma_{k}$ in the features hyper-space (with coordinates $\mathbf{x}_{\mathbf{k}}$ ), its margin is defined as the euclidean distance between the point and the hyper-plane:

$$
K M_{k}=d\left(\sigma_{k}, \epsilon\right)=\frac{\alpha+\boldsymbol{\beta} \mathbf{x}_{\mathbf{k}}}{\sqrt{\boldsymbol{\beta} \boldsymbol{\beta}^{T}}} .
$$

Combining equation (2) and (5) it is possible to relate the probability of the knock event to the measured margin:

$$
P\left(y=1 \mid K M_{k}\right)=\frac{e^{K M_{k} \sqrt{\boldsymbol{\beta}^{T}}}}{1+e^{K M_{k} \sqrt{\boldsymbol{\beta} \boldsymbol{\beta}^{T}}}}
$$

To successfully accomplish the modelling goal two operations are required. The first is the correct choice of the attributes $\mathbf{x}$, which will be addressed in the next Section. Once the features have been selected, a tuning procedure for the model parameters $\alpha$ and $\boldsymbol{\beta}$ is needed. Despite its appealing linear regression interpretation, practically, the procedure to identify such parameters is not based on a least-square fitting of the log-odds, but rather on the conditional likelihood $\mathcal{L}$ maximization defined as:

$$
\mathcal{L}_{\alpha, \beta}=\mathcal{P}\left(y_{1}, \ldots, y_{n} \mid \mathbf{x}_{\mathbf{1}}, \ldots, \mathbf{x}_{\mathbf{n}}\right)=\prod_{k=1}^{n} \frac{e^{\left(\alpha+\boldsymbol{\beta} \mathbf{x}_{\mathbf{k}}\right) y_{k}}}{1+e^{\left(\alpha+\boldsymbol{\beta} \mathbf{x}_{\mathbf{k}}\right)}},
$$

which is a nonlinear function of $\alpha$ and $\boldsymbol{\beta}$. Several iterative methods have been proposed to solve this problem and readyto-use implementations of such algorithms are the standard equipment of statistical data analysis software; in this context the Matlab glmfit function has been exploited.

Figure 4 provides an example of the application of the proposed modelling approach to a small subset of data. These data are taken in an operating point with constant speed, inlet air temperature and air-to-fuel ratio. In order to induce different knock levels the spark advance is changed from 20 to 35 degrees before top dead centre. As an example, following the suggestion of Lezius et alt. [18], the maximum cycle pressure has been used as cycle attribute $x(k)=\mathrm{p}_{\max }(k)$. The pressure values have been grouped in $j=20$ intervals. In the upper panel, within each group, both event frequencies

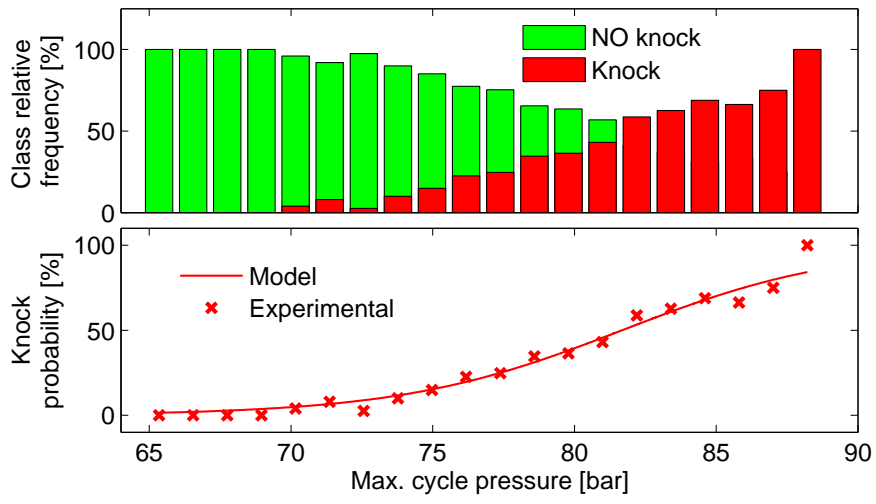

Fig. 4. Example of logistic regression modelling: the upper plot shows the relative knock/not knock event frequency within each peak pressure group; the lower plot shows the modelled and the experimental knock probability $P(y=1)$. (expressed in percentage) are shown:

$$
F_{1, j}=\frac{N_{1, j}}{N_{t o t, j}} \cdot 100 \quad \text { and } \quad F_{0, j}=\frac{N_{0, j}}{N_{t o t, j}} \cdot 100,
$$

where $N_{1, j}$ and $N_{0, j}$ are, respectively, the number of observed knocking and not-knocking cycles over the total $N_{t o t, j}$ number laying in the $j$-th group. In the lower panel the modelled (2) and experimental probability $\left(F_{1, j}\right.$, according to the frequentist probability interpretation) are shown.

Since in the proposed case the feature space is monodimensional, the classification hyper-plane becomes the scalar value $\bar{x}$, defined by:

$$
\bar{x}: \log \Lambda(\bar{x})=0 \approx \bar{x}=82.5 \text { [bar }] .
$$

In order to focus on the knock estimation, the cycle-domain modelling results are reported in Figure 5, for the same dataset previously considered. In the upper (first) panel the applied spark advance angle is shown: each operating point is characterized by a constant SA value. In the second plot the value of the maximum cycle pressure is reported, along with the classification threshold $\bar{x}$; the knock margin is proportional to the quantity $x(k)-\bar{x}$. In the same plot red crosses and green dots are used to mark if, for the corresponding cycle, knock has been measured $(y(k)=1)$ or not $(y(k)=0)$; finally note that, according to the classification rule:

$$
\begin{aligned}
& \hat{y}(k)=1 \text { if } \quad K M(k) \geq 0 \\
& \hat{y}(k)=0 \text { if } \quad K M(k)<0
\end{aligned},
$$

the expected model output $\hat{y}$ can be computed: thus for each point that lies above the threshold knock is expected; the opposite for all the points below the black dashed line. In the bottom panel the estimated model probability (2) is reported and, along with it, the measured knock probability, computed as (6) where the index $j$ refers to each constant SA operating point.

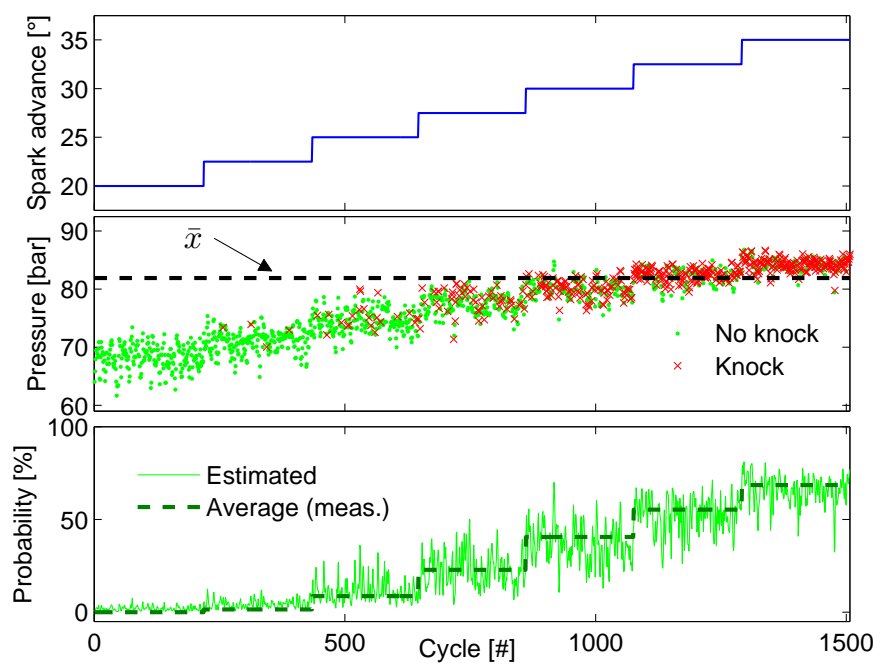

Fig. 5. Cycle-domain knock estimation. Upper plot shows the applied crank angle; in the middle plot the cycle feature $x(k)$ along with the classification threshold $\bar{x}$ is depicted; in the lower plot the estimated and the measured (of each $\mathrm{OP}$ ) probability. 


\section{B. Assessment of estimation quality}

Figure 5 allows one to stress another peculiarity of the proposed knock margin estimation approach. Indeed, unlike the normal regression problems, since the measured variable is dichotomous it is not convenient to define an error-like quantity to evaluate the estimation performances. The quality of the knock margin estimation should be evaluated comparing model output in terms of estimated probability: intuitively, a possibility is to compare the measured and the expected knock probability, as shown in the lower plot.

Traditionally, see [12], [22], two approaches have been used to assess model quality. In this work, both approaches are used to ensure the most comprehensive evaluation of the modelling performance.

On the one hand pseudo- $R^{2}$ indexes have been proposed, that describe the predicting power of the identified model compared to the predicting power of a null model i.e. when only the intercept $(\alpha)$ is present. The other approach considers the calibration of the model, that is to say, it judges the agreement between observed and expected outcomes. In this work, both approaches are used to ensure the most comprehensive evaluation of the estimation performance.

Among the several pseudo- $R^{2}$ indexes (see [22] for a detailed comparison) the one employed here is the $R_{L}^{2}$ index described in [21]:

$$
R_{L}^{2}=1-\frac{\log \mathcal{L}_{\mathcal{M}}}{\log \mathcal{L}_{0}}
$$

where $\mathcal{L}_{M}$ is the likelihood of the identified model, whereas $\mathcal{L}_{0}$ is the likelihood of the null model. Conceptually similar to the $R^{2}$ index of linear regression, its appeal is due to the direct relationship with the quantity i.e. the likelihood that is used to identify the model parameters.

In order to evaluate the agreement between the observed and estimated data, various approaches can be followed, see [11]. In the present work the method proposed by Hosmer and Lemeshow [12] is used. Groups are built first, such that the same number of observed events are present in each, followed by the computation of a Pearson's residual based statistic:

$$
\hat{C}=\sum_{n=1}^{n_{g}}\left(\frac{\left(O_{1}-E_{1}\right)^{2}}{E_{1}}+\frac{\left(O_{0}-E_{0}\right)^{2}}{E_{0}}\right) .
$$

Equation (8) can be easily interpreted as a sum-of-error like index, where for each class (0: not-knocking, 1: knocking) the error is computed as difference between the expected $(E)$ and the observed $(O)$ event occurrences normalized with respect to the expected frequencies. Regarding the number of groups in which the attributes are divided, a fixed value of $n_{g}=20$ groups is used from now on, mainly for graphical yield. However, the results of the following sections, based on a relative comparison of fit indexes, have been tested with different groupings leading to the same conclusions.

\section{KNOCK MARGIN MODELS}

In the previous Section the model-based estimation approach has been set. However a still open point is the choice of the model input, i.e. the attributes that are the representative features of an engine cycle is discussed. Two main problems arise. First of all, all or most of the knock influencing factors have to be included directly or indirectly in the model. The next question is how the pressure trace should be treated: Figure 2 shows how pressure profiles carry the knock margin information but, given the complex shape of a pressure profile, the way in which such information is to be extracted from the pressure profile is not straightforward. In the following, two competing approaches are presented.

\section{A. Physical approach}

The most straightforward way to build knock representative features is based on earlier studies on physical knock mechanisms. This approach is labelled here as physical.

Given in-cylinder conditions a well-known way to model knock occurrence is the so-called knock integral first introduced by Livengood and $\mathrm{Wu}$ [20], which is widely employed in engine knock research. The idea is to lump all the complex chemical reactions that eventually lead to end-gas self-ignition into a single Arrhenius-like formulation:

$$
\tau=C_{1} p^{C_{2}} e^{\frac{C_{3}}{T}},
$$

where $\tau$ is the so-called self-ignition time, which is the time required by the mixture to self-ignite at certain pressure and temperature conditions. Equation (9) represents a semiphysical chemical model since it keeps the physical structure of the reactions in the nonlinear combination of pressure $p$ and temperature $T$, whereas its coefficients are determined experimentally. To cope with the non-constant pressure and temperature conditions in the combustion chamber during the evolution of the cycle, an integral formulation of that equation has been proposed. Expressed in the crank angle domain, it states that knock occurs when:

$\frac{1}{6 \omega} \int_{\theta_{0}}^{\theta_{k n}} \frac{1}{\tau(\theta)} d \theta=1 \rightarrow \frac{1}{6 \omega C_{1}} \int_{\theta_{0}}^{\theta_{k n}} p(\theta)^{-C_{2}} e^{-\frac{C_{3}}{T(\theta)}} d \theta=1$,

where $\theta_{0}$ is the angle when the reaction starts (usually the intake valve closing time, IVC), $\theta_{k n}$ is the self-ignition angle and $\omega$ is the engine speed in rpm

Given the in-cylinder conditions of a $k$-th cycle $p_{k}(\theta)$ and $T_{k}(\theta)$, with equation (10) the expected self-ignition angle $\hat{\theta}_{k n, k}$ can be computed. Intuitively, if the predicted knock angle oversteps the end-of-combustion angle no knock can occur because all the end-gas has been burnt. The knock margin thus could be defined as the distance from the endof-combustion angle. Several researchers have generalized this concept defining a knock margin as the distance from a socalled critical angle $\theta_{c}$ :

$$
K M_{k}^{p h y}=\hat{\theta}_{k n, k}-\theta_{c} .
$$

There is no unique definition for the critical angle; usually each author relates it to a specific experimental evidence, see e.g. [5], [4]. In this work the critical angle is chosen to be the one maximizing the knock margin estimation performance.

The peculiar feature of the physics-based approach is the fact that the knock margin (11) is not related to any "geometric distance" concept, since it is based merely on 
physical considerations. In this perspective, the logistic regression downgrades to the single-input case, i.e. the classification hyper-plane becomes a scalar threshold which defines the equal probability of knock/not knock events. However, the logistic regression is still essential in order to assign the corresponding knock probability to each knock margin value.

To apply the proposed approach, the temperature $T(\theta)$ of the end-gas is needed, along with suitable values of the parameters $C_{1}, C_{2}$ and $C_{3}$ of the self-ignition time formula (9). Although measurement of the required temperature is not available, a well established procedure to estimate its value can be used (see e.g. [10]).

To estimate the parameters in equation (9), an optimization problem has been solved; this approach has already been proposed in literature, see [4], [26]. The values of $C_{1}, C_{2}$ and $C_{3}$ are found by minimizing the following cost function:

$J\left(C_{1}, C_{2}, C_{3}\right)=\sum_{j=1}^{n_{c}}\left(1-\frac{1}{6 \omega_{j} C_{1}} \int_{\theta_{0, j}}^{\theta_{k n, j}} p_{j}(\theta)^{-C_{2}} e^{-\frac{C_{3}}{T_{j}(\theta)}} d \theta\right)^{2}$,

where $n_{c}$ is the number of cycles considered. Since the experimental knock onset crank angle $\theta_{k n, j}$ is required, only measured knocking cycles are used for the optimization problem.

The nonlinear optimization problem is very sensitive to initial conditions. Thus it has been solved using a brute force Particle Swarm Optimization (PSO) algorithm available for Matlab [3]. The resulting values of the coefficients are listed in Table I, along with other values that can be found in literature. Whereas there is a general agreement about the

TABLE I

COMPARISON OF SELF-IGNITION MODEL (9) COEFFICIENT VALUES.

\begin{tabular}{l|ccc} 
& $C_{1}$ & $C_{2}$ & $C_{3}$ \\
present work & 0.344 & -1.84 & 1524 \\
Elmqvist et al. [4] & 0.021 & -1.7 & 3800 \\
Douaud and Eyzat [2] & 0.018 & -1.7 & 3800
\end{tabular}

second coefficient $C_{2}$, different values result for $C_{1}$ and $C_{3}$. Figure 6 shows the cost function $J$ for different values of $C_{1}$ and $C_{3}$, while keeping $C_{2}$ constant. Clearly there is no welldefined unique minimum. This explains why combinations of $C_{1}$ and $C_{3}$ can yield a satisfactory performance and why the choice of initial conditions is crucial if standard gradient-based optimization algorithms are used in this context. An identical behaviour has already been pointed out in [4], which is the only literature paper that clearly states the difficulty in finding self-ignition parameters.

Despite the apparent over-parametrization of the optimization problem the resulting choice of coefficients yields a satisfactory knock modelling performance, showing a good agreement between the measured and the estimated knock onset crank angles (KOCA). Figure 7 shows the KOCA estimation error for the training and validation set of knocking

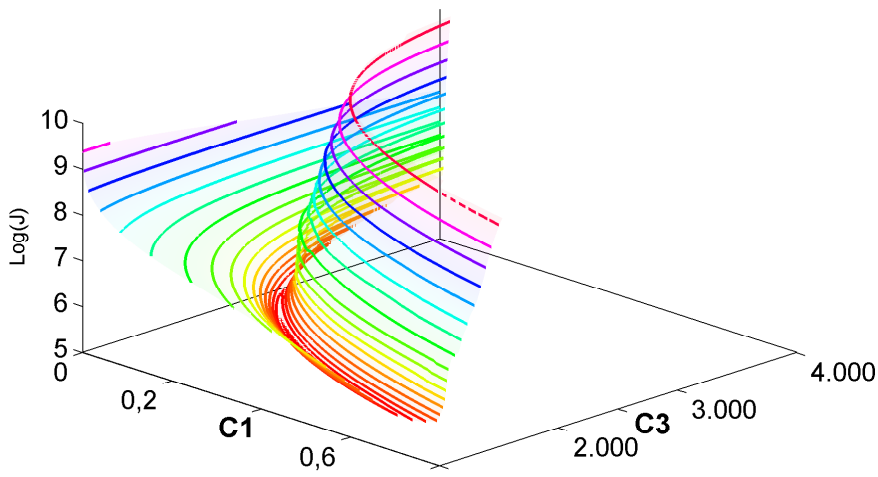

Fig. 6. Cost function (IV-A) for different values of $C_{1}$ and $C_{3}$. cycles, with datasets that have been generated by randomly grouping knocking cycles for all the engine operating points considered.
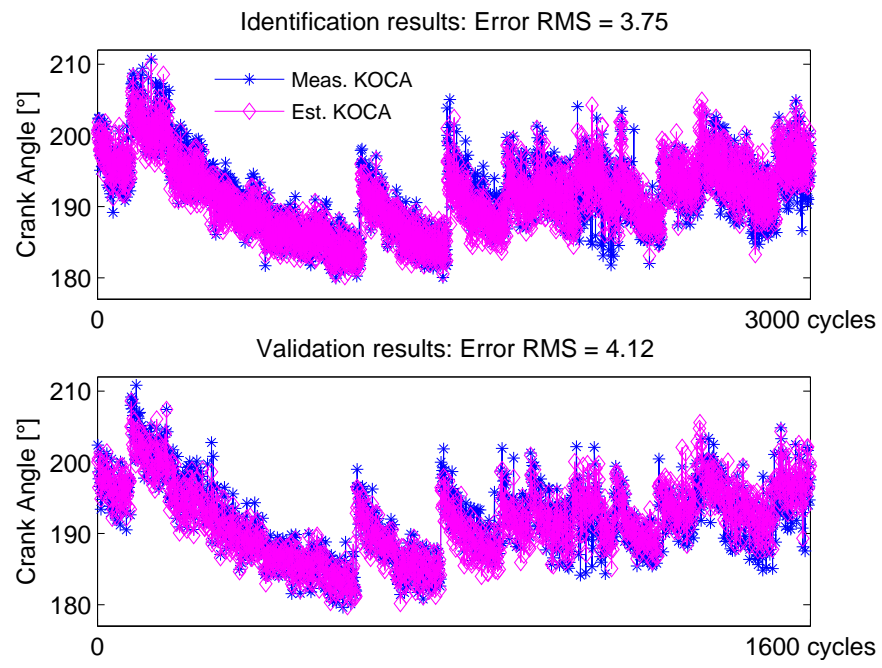

Fig. 7. Comparison between measured and estimated KOCA with the knock integral model (10). The upper panel shows training data results; the lower panel the validation performance.

\section{B. Grey-box approach}

The alternative solution for knock modelling exploits the multi-variable capability of the logistic regression algorithm. Instead of combining different measurements within the physics-based structure (10) and directly building the knock margin, more attributes are used to identify a multi-variable logistic regression model. Since the model is built combining physically relevant variables within a non-physical - even though pre-assigned - linear structure, this procedure here is called grey-box.

The choice of the attributes, i.e. the values and measurements that represent each engine cycle, becomes the crucial point of the proposed approach. As suggested by physical principles at least three quantities are necessary, related to engine speed, in-cylinder pressure and temperature, respectively.

Engine speed is an available signal, thus its average value during each cycle $\bar{\omega}$ can be directly used as an attribute.

To include in-cylinder temperature information the following facts have to be considered. First, as discussed in [10], 
only high pressures and temperatures contribute to the self ignition of the end-gas; thus, only the combustion phase can be considered relevant. Assuming the adiabatic compression of the end-gas during combustion, its temperature evolution can be computed using the following expression:

$$
T(\theta)=T\left(\theta_{I V C}\right)\left(\frac{P(\theta)}{P\left(\theta_{I V C}\right)}\right)^{1-\frac{1}{\gamma}} \quad, \quad \theta>\theta_{S I} .
$$

The inspection of the proposed equation suggests that the incylinder temperature information is fully known if the pressure profile and the temperature of the mixture at intake valve closing $(I V C)$ angle are available. The variable $T_{I V C}$ is well described by the intake manifold temperature $T_{i m}$ that can be measured by a series production sensor. Thus, this value has been selected as the second feature describing an engine cycle.

To carry the required pressure information, the in-cylinder pressure signal is employed. This sensor measures the pressure profile with a crank angle resolution of 0.75 , consisting of $360 \cdot 0.75=480$ relevant points for each cycle (in a fourstroke engine each cycle covers 720 degrees, only 360 of which are of interest for the combustion). In principle, all of these 480 variables could be used as features representing pressure. However, due to the fact that in model identification the variance of the estimation strongly depends on the number of inputs [15], the proposed approach is poorly reliable in practice. This is a common problem when dealing with modelling internal combustion engines. To cope with it in literature some solutions can be found. Among the simplest, one option is to synthesize the entire pressure profile with some selected values, such as the maximum pressure value and its crank angle location or the maximum pressure gradient and the mean effective pressure. However, such an approach is limited by the excessive loss of information occurring when the pressure profile is described simply with these values, that are too local or too mean.

To overcome this limitation a Principal Component Analysis (PCA), also known as Karhunen-Love transform [14], approach is adopted in this paper. This solution has shown promising results when dealing with control and estimation issues of internal combustion engines (see [23], [6], [31]). PCA aims at dimensionality reduction or feature extraction. Recalling, as an example, the upper panel of Figure 3 one can realize that although different, all the pressure traces clearly show an underlying common trend (the bell shape). In this context the PCA approach aims at extracting such common information, thus possibly reducing the number of parameters needed to represent each pressure profile.

In order to do so, the pressure matrix $P_{480 \times N}$ (where $\mathrm{N}$ is the number of engine cycles considered, $N>480$ ) that collects all the pressure profiles is decomposed using the singular value decomposition (SVD):

$$
P=U \Sigma V^{T}
$$

where $\mathrm{U}$ and $\mathrm{V}$ are orthogonal matrices, while $\Sigma$ is a diagonal matrix with the non-zero terms $\sigma_{i}, i=1, \ldots, 480$. The matrix U contains 480 orthogonal vectors of length 480 , the so-called eigenpressures $\bar{p}_{i}(\theta)$ where the $\theta$ dependency has been added to stress the crank-angle domain of the pressure profile. A given measured pressure trace $p_{k}(\theta)$ can be thus expressed as a linear combination of the first 480 eigenpressures:

$$
p_{k}(\theta)=\sum_{i=1}^{m} \gamma_{i, k} \bar{p}_{i}(\theta), m=1, \ldots, 480,
$$

where the coefficients $\gamma_{i, k}$ are defined as the projection of the pressure profile on the respective $i$-th eigenpressure. Such projection is computed by applying the inner product:

$$
\gamma_{i, k}=<p_{k}(\theta), \bar{p}_{i}(\theta)>\text {. }
$$

Equation (12) states that a given pressure profile (consisting of 480 variables) can be described with $m \leq 480$ coefficients. The more features are used, the smaller the error between the measured and the reconstructed profile is, hence the loss of information in representing the measured profile with the corresponding $\gamma_{i, k}, i=1, \ldots, m$ coefficients. Considering the nature of SVD, the singular values are sorted in a decreasing order in the matrix $\Sigma$ according to the relevance of each eigenpressure in the pressure profile reconstruction. To obtain a satisfactory reconstruction only the first 3 eigenpressures are needed: the left panel of Figure 8 shows the first 5 singular values, evidencing this fact. Figure 9 shows the comparison between a measured and its reconstructed profile using the first 3 eigenpressures that are shown in the right panel of Figure 8 ; the lower plot shows the reconstruction error.
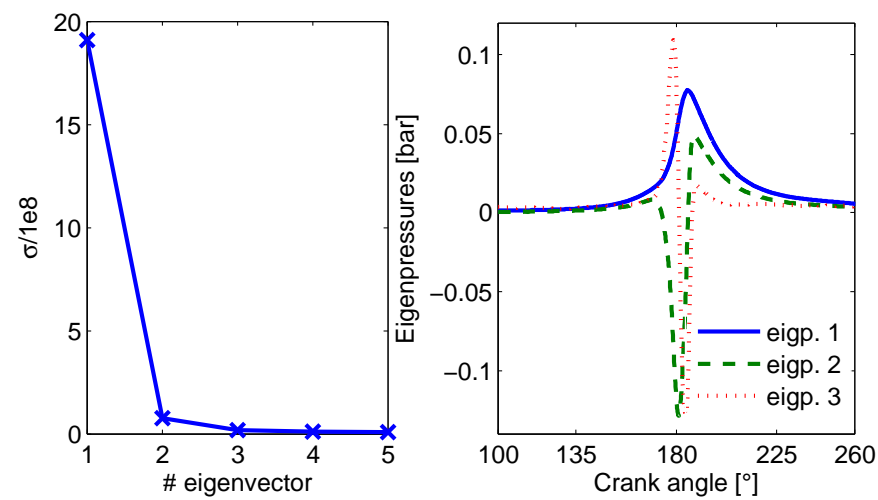

Fig. 8. Singular values (left panel) and first 3 corresponding eigenpressures (right panel).

The first 3 PCA coefficients $\gamma_{1}, \gamma_{2}, \gamma_{3}$ are thus considered as representative of a generic pressure profile and are used as features to carry the in-cylinder pressure information needed for the logistic regression model. The benefits of the PCA approach can be appreciated noting the significant reduction of features used to describe the in-cylinder pressure profile, namely from 480 to 3 .

In summary, the $k$-th engine cycle is defined by the following set of attributes $\mathbf{x}_{\mathbf{k}}^{\mathbf{g b}}$ :

$$
\mathbf{x}_{\mathbf{k}}^{\mathbf{g b}}=\left[\begin{array}{lllll}
\gamma_{1, k} & \gamma_{2, k} & \gamma_{3, k} & T_{i m, k} & \bar{\omega}_{k}
\end{array}\right] .
$$

Once the logistic model coefficients $\alpha_{g b}$ and $\boldsymbol{\beta}_{g b}$ are identified, the knock margin is defined as the euclidean distance defined in (5).

$$
K M_{k}^{g b}=d\left(\sigma_{k}^{g b}, \epsilon_{g b}\right)
$$



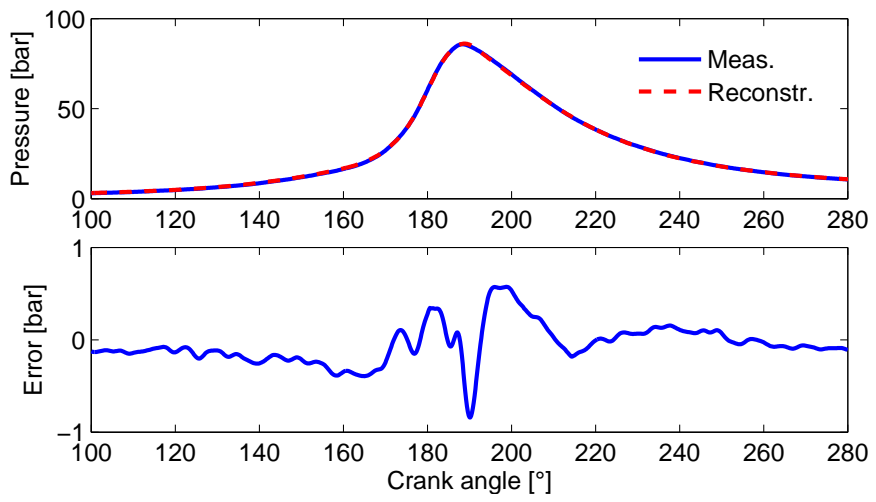

Fig. 9. Comparison between measured and reconstructed pressure using the first 3 features (upper plot). Pressure error in the lower plot.

\section{EXPERIMENTAL RESULTS}

\section{A. Experimental set-up}

Measurements were conducted on a fully equipped test bench consisting of an SI engine and an electric brake. The 0.75 litres engine features 2 parallel twin cylinders, a single stage turbocharger and port fuel injection for a maximum power of $61 \mathrm{~kW}$ at $6000 \mathrm{rpm}$ and a maximum torque of $131 \mathrm{Nm}$ at $3000 \mathrm{rpm}$. The electric brake has been used in the speedcontrol mode, which permits the engine to be tested at different engine speed. Each component has a devoted dSpace ${ }^{\circledR}$ rapid prototyping system that handles the engine and brake control and the logging tasks. Moreover, in order to sample the incylinder signals at the adequate rate, a Teledyne LeCroy ${ }^{\circledR}$ WaveSurfer oscilloscope capable of a sampling frequency of up to $2.5 \mathrm{GHz}$ has been used.

\section{B. Results}

The comparison among different knock models is based on data collected from different operating points where the engine runs in a standard way, which means that the injection control is active, aiming at regulating the air-to-fuel ratio $(\lambda)$ to the stoichiometric value $(\lambda=1)$. It is well known (e.g. [33]) that the air-to-fuel ratio is another factor that mainly influences knock. Given the presence of a $\lambda$ controller, which keeps its value constant, such variable has not been included in the set of those used in the estimation. However, if needed, the estimators comparison could be easily extended in the following way: for the physical-based model the self-ignition delay (9) could be modified according to the expressions suggested by some authors, such as in [27], [29]. On the other side, the average-cycle measured AFR value could be simply added to the features vector (13).

To check the influence of various factors on knock tendency, experimental tests have been carried out as follows:

1) Different speeds, ranging from 1800 to $3000 \mathrm{rpm}$ have been tested.

2) For each engine speed, the engine load has been set to produce a torque of around $100 \mathrm{Nm}$; the spark was then advanced w.r.t. the optimal operating point value - as shown in Figure 5 - thus reducing the load. Tests were interrupted when the conditions became too extreme, e.g. warning level of knock rate or intensity were reached, or the pressure peaks became too high.

3) For the same engine speed, the intake manifold temperature was increased. To do so the inter-cooler cooling water flow was reduced, since the ambient cell temperature could not be changed. Due to the higher temperatures, the $100 \mathrm{Nm}$ engine output torque could not be always reached.

4) For each operating point described, steady-state conditions were waited before data acquisition begun and an average of 375 cycles were collected.

Note that the experimental main focus was placed on the most significant engine knocking operating region: for this reason very high speed and low load conditions were not explored. Given the inner linearity hypothesis of the PCA, limiting the engine operations to the edge-knocking conditions helps to provide better estimation results. Nevertheless, as will be shown in the following, the explored area features a ratio of knocking cycles from 0 to almost $50 \%$ which, in a knocking control perspective is a more than adequate range (usually the desired knock ratio is kept below 5\%, see e.g. [25]).

Knock margin estimation performances with the physical and grey-box approaches are shown in Figure 10. To allow an easier quantitative comparison, the respecting knock margins (11) and (14) have been normalized. The upper panels show the relative event frequencies as a function of the knock margin value for each model. Generally, an increasing knock tendency can be observed for both approaches; however for the grey-box model the increase of knock tendency with the knock margin is more relevant and it is monotonic.

The left lower plots show the $K M$ values as function of the engine cycles: for the sake of clarity, knocking and not knocking events have been marked with different colours (red crosses and green dots, respectively). With the physical approach knocking and not knocking cycles are characterized by similar margin values; opposite, the grey-box yields a clearer distinction between the two situations. In the same plots, with the grey-box approach a certain grouping of $K M$ values can be noticed. Considering the type of tests that where performed (in each OP the spark advance was changed to achieve an higher knock rate until a limit value was reached) it is easy to understand that each group refer to a specific OP. Looking at the physics-based model such data grouping vanishes, highlighting the poor discriminative power and the loss of information in using such approach to estimate the knock rate.

Finally, the right lower plots depict the observed and the estimated knock probabilities, as a function of the knock margin value. With the fit quality indexes reported in Table II a quantitative comparison between the two approaches can be set. If one considers the $\hat{C}$ index, there is not much difference between the grey-box and the physics-based estimation (though the lower value of $\hat{C}$ of the former indicates a slightly better agreement between the observed and the estimated probabilities). However the most significant difference is carried by the $R_{L}^{2}$ index, which is significantly higher for the grey-box case. This means that the $K M$ computed with such method carries much more information about the 

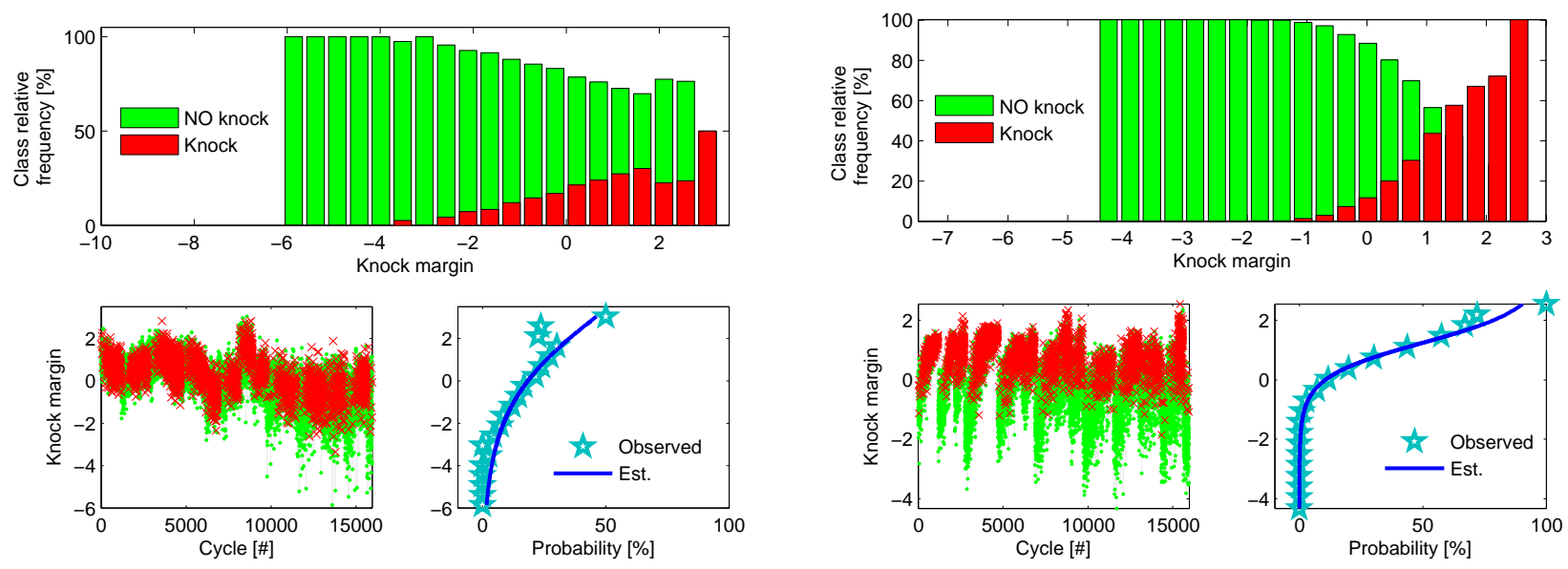

Fig. 10. Knock margin models comparison: left plot shows the physics-based knock margin performance. On the right side the performance of the gray-box knock margin model are presented.

knock rate than with the physics-based. Looking at the right lower plots this fact is shown by the steeper change of knock probability - w.r.t the knock margin - achieved with the grey-box estimation approach. The worse performance of

TABLE II

MODELS PERFORMANCE COMPARISON.

\begin{tabular}{l|cc} 
Index & $R_{L}^{2}$ & $\hat{C}$ \\
Physic-based & 0.025 & 120.8 \\
grey-box & 0.23 & 112.3
\end{tabular}

the physics-based model can be explained noticing that in the identification stage only part of the knock information was used. Indeed, for the physical approach the definition of the knock margin requires the identification of the KOCA model (10), which uses only knocking cycles (the only ones for which an experimental KOCA can be defined). In this perspective, all the information provided by the not-knocking cycles is ignored. In contrast, the grey-box model aims at the best possible classification performance and thus it equally exploits the information carried by experimental data, both knocking and not-knocking cycles.

\section{CONCLUSION}

The knock margin control strategy is a promising approach to enhance the performance of knock control algorithms. Given measured values of an engine cycle, the knock margin quantifies the distance from a knocking conditions. In the present work, thanks to the availability of in-cylinder pressure sensors, the knock margin concept has been developed with a systematic approach. Its effectiveness is validated by experimental data results.

In the first part of the paper, the model-based knock margin estimator is set. A logistic-regression model proves useful for two reasons: on the one side its geometrical interpretation helps a handy definition of the knock margin concept, computed as geometric distance between point an plane in an hyper-space. On the other its useful statistical interpretation allows knock margin values to be related to the control target knock probability.

In the second part of the work two competing approaches to build proper regressors have been presented. The first one combines the engine measures - in-cylinder pressure, temperature and engine speed - in a non-linear physical-driven way, in order to derive a knock margin variable which is then used to model knock probability. The second approach, called grey-box, exploits the multivariate capability of the logistic regression algorithm combining various inputs, that represent the information carried by measured signals, to model knock probability. In order to keep the size of the engine cycle feature array as small as possible, aiming at the best modelling performance, physical considerations and a principal component analysis (PCA) have been employed.

Experimental results show that the grey-box approach leads to a better estimation performance, using only three pressure features, the intake manifold temperature and engine speed measures.

Engine knock can be caused by many sources and some of them could not be easily measured e.g. fuel quality, hotspots. A possible improvement of the proposed approach would deal with the development of an adaptive extension of the knock margin estimator, capable of adapting its parameters w.r.t. the measured knock rate, in order to cope with changes in those unmeasured factor. It should be noted that this issue affects both the physical and the grey-box approaches; besides its better estimation performances, given its easier structure and procedure for parameter estimation, the grey-box model is likely the better candidate for the development of such extension.

Given the promising estimation results, future work will also deal with the use of the knock margin in closed loop knock control strategies.

\section{REFERENCES}

[1] A. di Gaeta, V. Giglio, G. Police, and N. Rispoli, "Modeling of incylinder pressure oscillations under knocking conditions: A general 
approach based on the damped wave equation," Fuel, vol. 104, pp. 230 243, 2013.

[2] A. M. Douaud and P. Eyzat, "Four-Octane-Number Method for Predicting the Anti-Knock Behavior of Fuels and Engines," SAE Technical Paper series, no. 780080, 1978.

[3] S. Ebbesen, P. Kiwitz, and L. Guzzella, "A generic particle swarm optimization Matlab function," in American Control Conference (ACC), Montreal, 2012, pp. 1519-1524.

[4] C. Elmqvist, F. Lindström, H.-E. A ngström, B. Grandin, and G. Kalghatgi, "Optimizing Engine Concepts by Using a Simple Model for Knock Prediction," SAE Technical Paper series, no. 2003-01-3123, 2003.

[5] S. Fontanesi and S. Paltrinieri, "Knock Tendency Prediction in a High Performance Engine Using LES and Tabulated Chemistry," SAE International Journal of Fuels and Lubricants, vol. 6(1), pp. 98-118, 2013.

[6] S. Formentin, M. Corno, H. Waschl, D. Alberer, and S. M. Savaresi, "NOx Estimation in Diesel Engines via In-Cylinder Pressure Measurement," IEEE Transactions on Control Systems Technology, vol. 22, no. 1, pp. 396-403, 2014

[7] A. Gogan, B. Sundén, L. Montorsi, S. S. Ahmedand, and F. Mauss, "Knock Modeling: an Integrated Tool for Detailed Chemistry and Engine Cycle Simulation," SAE Technical Paper series, no. 2003-01-3122, 2003.

[8] I. Haskara and J. Winkelman, "Stochastic limit control and its application to spark limit control using ionization feedback," in Proceedings of the 2005 American Control Conference. IEEE, 2005, pp. 5027-5034.

[9] E. Hellstrom and A. G. Stefanopoulou, "Modeling cyclic dispersion in autoignition combustion," in IEEE Conference on Decision and Control and European Control Conference. Orlando, FL: IEEE, 2011, pp. 6834 6839.

[10] J. Heywood, Internal combustion engine fundamentals. McGraw-Hill, 1988.

[11] D. W. Hosmer, T. Hosmer, S. Le Cessie, and S. Lemeshow, "A comparison of goodness-of-fit tests for the logistic regression model." Statistics in Medicine, vol. 16, no. 9, pp. 965-980, 1997.

[12] D. W. Hosmer, S. Lemeshow, and R. X. Sturdivant, Applied Logistic Regression, 3rd ed. Hoboken, NJ, USA: John Wiley \& Sons, Inc., 2013.

[13] A. K. Jain, R. P. Duin, and M. Jianchang, "Statistical pattern recognition: a review," IEEE Transactions on Pattern Analysis and Machine Intelligence, vol. 22, no. 1, pp. 4-37, 2000.

[14] I. T. Jolliffe, Principal Component Analysis, 2nd edition, 2nd ed., ser. Springer Series in Statistics. Springer, 2002.

[15] A. Juditsky, H. k. Hjalmarsson, A. Benveniste, B. Delyon, L. Ljung, J. SjÖberg, and Q. Zhang, "Nonlinear black-box models in system identification: Mathematical foundations," Automatica, vol. 31, no. 12, pp. 1725-1750, 1995.

[16] S. Ker, F. Bonnardot, and L. Duval, "Algorithm comparison for real time knock detection," in ICASSP, IEEE International Conference on Acoustics, Speech and Signal Processing - Proceedings, vol. 2, 2007.

[17] U. Kiencke and L. Nielsen, Automotive Control Systems for Engine, Driveline, and Vehicle, 2nd ed. Springer, 2005.

[18] U. Lezius, M. Schultalbers, W. Drewelow, and B. Lampe, "Improvements in knock control," in 2007 Mediterranean Conference on Control \& Automation. Athens: IEEE, 2007, pp. 1-5.

[19] Z. Liu and R. Chen, "A Zero-Dimensional Combustion Model with Reduced Kinetics for SI Engine Knock Simulation," Combustion Science and Technology, vol. 181, no. 6, pp. 828-852, 2009.

[20] J. Livengood and P. Wu, "Correlation of autoignition phenomena in internal combustion engines and rapid compression machines," Symposium (International) on Combustion, vol. 5, no. 1, pp. 347-356, 1955.

[21] D. McFadden, "Conditional logit analysis of qualitative choice behavior," in Frontiers in econometrics, P. Zarembka, Ed. Academic Press, 1974, pp. 105-142.

[22] S. Menard, "Coefficients of determination for multiple logistic regression analysis," The American Statistician, vol. 54, no. 1, pp. 17-24, 2000.

[23] D. Moser, S. Hahn, H. Waschl, and L. del Re, "Torque control of a diesel engine by an eigenpressure based approach," in European Control Conference (ECC), 2013, Zürich, 2013, pp. 434-439.

[24] J. C. Peyton Jones, J. Frey, and K. R. Muske, "A Fast-Acting Stochastic Approach to Knock Control," in Proceedings of the 2009 IFAC Workshop on Engine and Powertrain Control, Simulation and Modeling, ECoSM'09, A. Sciarretta, Ed., Rueil-Malmaison, 2009, pp. 16-23.

[25] J. C. Peyton Jones, J. M. Spelina, and J. Frey, "Likelihood-Based Control of Engine Knock," IEEE Transactions on Control Systems Technology, vol. 21, no. 6, pp. 2169-2180, 2013.
[26] S. Soylu and J. Van Gerpen, "Development of an autoignition submodel for natural gas engines," Fuel, vol. 82, no. 14, pp. 1699-1707, 2003.

[27] I. Z. Syed, A. Mukherjee, and J. D. Naber, "Numerical Simulation of Autoignition of Gasoline-Ethanol/Air Mixtures under Different Conditions of Pressure, Temperature, Dilution, and Equivalence Ratio," SAE Technical Paper series, no. 2011-01-0341, 2011.

[28] S. Tielong and O. Yasufumi, "Experimental Analysis and ControlOriented Modeling for Cyclic Variation of Cylinder Pressure in IC Engines," in 2007 Chinese Control Conference. Hunan: IEEE, 2007, pp. 613-617.

[29] J. Vancoillie, L. Sileghem, and S. Verhelst, "Development and Validation of a Knock Prediction Model for Methanol-Fuelled SI Engines," SAE Technical Paper series, no. 2013-01-1312, 2013.

[30] S. Yue and P. Li, "Automatic Knock Control System," in Fifth World Congress on Intelligent Control and Automation, WCICA 2004. Hangzhou, 2004, pp. 2464-2466.

[31] Y. Zhan, Z. Shi, and M. Liu, "The Application of Support Vector Machines (SVM) to Fault Diagnosis of Marine Main Engine Cylinder Cover," in IECON 2007 - 33rd Annual Conference of the IEEE Industrial Electronics Society. IEEE, 2007, pp. 3018-3022.

[32] K. Zhang, G. G. Li, F. C. Xu, and Y. Li, "Probability distribution of knock factor and knock-band method for knock detection," in 2010 International Conference on Mechanic Automation and Control Engineering, MACE2010, 2010, pp. 6049-6053.

[33] X. Zhen, Y. Wang, S. Xu, Y. Zhu, C. Tao, T. Xu, and M. Song, "The engine knock analysis - An overview," Applied Energy, vol. 92, pp. 628-636, 2012.

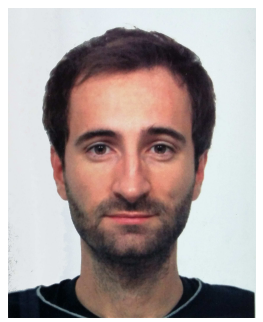

Giulio Panzani was born in Monza (Italy), in 1984. $\mathrm{He}$ received the Bachelor and Master of Science cum laude in Mechanical Engineering from the Politecnico di Milano in September 2005 and April 2008, respectively. In 2012 he earned the Ph.D. in Information engineering (system control specialization) from Politecnico di Milano.

He held post-doc positions at the University of Trento and at the Swiss Federal Institute of Technology of Zuerich (ETHZ). Since April 2015, he is assistant professor at Politecnico di Milano.

His main research interests include the analysis of dynamics, control design and estimation for two (and four) wheeled vehicles and internal combustion engine control.

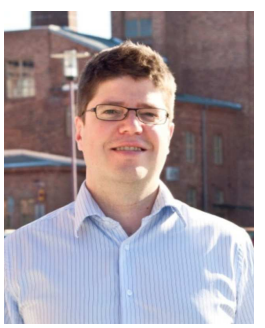

Fredrik Östman received his MSc. diploma in Computer Engineering and $\mathrm{PhD}$. diploma in System Engineering from Åbo Akademi University 2003 and 2009, respectively.

Since 2008, hes been working at Wärtsilä, a manufacturer of engines and other equipment for the marine and energy markets. Between 2010 and 2013, he headed the engine control system development team for 4-stroke engines, after which he continued with various management positions related to $R \& D$ of 4-stroke engines

Hes currently a senior project manager where hes amongst other things involved with university research activities. He holds over 10 patents and has authored 10 scientific journal and conference papers.

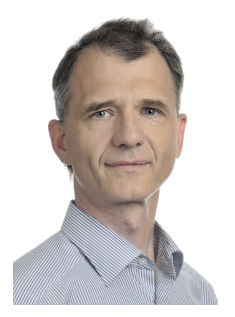

Christopher H. Onder is professor at the Institute for Dynamic Systems and Control ETH Zurich. He heads the Engine Systems Laboratory and holds a diploma and a doctoral degree in Mechanical Engineering from ETH Zurich.

$\mathrm{He}$ is the author and co-author of numerous articles and a book on modeling and control of engine systems. The list of his awards includes the BMW scientific award, the ETH medal, the Vincent Bendix award, the Crompton Lanchester Medal, and the Arch T. Colwell award. 